This is a postprint version of the following published document:

Valencia, J., Baselga, J., E. Pacios, I. \& F. Piérola, I. (2003). Compression elastic modulus of neutral and protonated poly( $\mathrm{N}$-vinylimidazole) hydrogels. Macromolecular Symposia. Functional Networks and Gels, 200 (1), pp. 235-242.

DOI: $10.1002 /$ masy.200351024

(C) Wiley, 2003 


\title{
Compression Elastic Modulus of Neutral and Protonated Poly(N-vinylimidazole) Hydrogels
}

\author{
Joaquín Valencia, ${ }^{1}$ Juan Baselga, ${ }^{2}$ Isabel E. Pacios, ${ }^{1}$ Inés F. Piérola* ${ }^{1}$ \\ ${ }^{1}$ Departamento de Ciencias y Técnicas Fisicoquímicas, Facultad de Ciencias, Universidad a \\ Distancia (UNED), 28040 Madrid, Spain \\ E-mail: ipierola@ccia.uned.es
}

${ }^{2}$ Instituto Tecnológico de Química y Materiales, Universidad Carlos III, 28911 Leganés, Spain E-mail: jbaselga@,ing.uc3m.es

Summary: The compression modulus of poly(N-vinylimidazole) (PVI) hydrogels synthesized by cross-linking polymerization in aqueous solution, was measured at room temperature in several related systems: i) just after polymerization, ii) swollen at equilibrium in deionized water, iii) swollen in $\mathrm{HCl}(\mathrm{aq})(\mathrm{pH}=2.5)$, iv) swollen in $\mathrm{HCl}(\mathrm{pH}=2.5)$ and $1 \mathrm{M} \mathrm{NaCl}(\mathrm{aq})$ solution and v) swollen in $\mathrm{H}_{2} \mathrm{SO}_{4}(\mathrm{pH}=2.5)(\mathrm{aq})$ solution. Samples of the

first and second groups are neutral whereas hydrogels of the other three groups are ionic because of protonation of basic imidazole groups. The experimental results were fitted with the

Erman-Monnerie theory, applied to compression measurements for the first time, to determine the phantom modulus, $\left[f_{\mathrm{ph}} *\right]$, and the parameter $\kappa_{\mathrm{G}}$ which measures the constraining role of entanglements on the fluctuations of chains between knots.

Keywords: compression; elastic modulus; hydrogels; ionic gels; swelling

\section{Introduction}

The cross-link density of hydrogels is difficult to estimate from its mechanical characterization because xerogels tend to be extremely rigid and swollen hydrogels very often lose the necessary mechanical integrity. ${ }^{[1-4]}$ Nevertheless, to understand the mechanical behaviour of neutral and ionic polymer hydrogels represents an interesting challenge with important implications in the daily increasing number of hydrogel uses. This will be the aim of this work.

Poly(N-vinylimidazole) hydrogels (PVI) are particularly interesting in this respect because basic imidazole rings become protonated in acid media, ${ }^{[5]}$ and as a consequence, with the same sample, neutral and ionic hydrogels are obtained by swelling in neutral or acid solutions. Protonated rings are randomly distributed along the polymer network to minimize the energy due to electrostatic interactions and this avoids the heterogeneity intrinsic to ionic cross-linked copolymers formed by a neutral monomer and a charge carrying co-monomer, due to their different reactivity ratios. ${ }^{[6]}$ 
The Erman-Monnerie theory ${ }^{[7-10]}$ was applied here to interpret the compression results of swollen PVI hydrogels. This theory was successfully applied to fit stress-strain data of swollen cross-linked polymers and it has the advantage over previous theories of using only two fitting parameters, the phantom modulus $\left[f_{\mathrm{ph}}{ }^{*}\right]$ and $\kappa_{\mathrm{G}}$, which measures the constraining role of entanglements.

\section{Experimental Section}

Poly(N-vinylimidazole) hydrogels were synthesized by radical cross-linking polymerization of $\mathrm{N}$-vinylimidazole (VI) and N,N'-methylene-bis-acrylamide (BA) in aqueous solution, with AIBN $\left(6 \times 10^{-3} \mathrm{M}\right)$ as initiator. N-vinylimidazole purchased from Aldrich, was distilled under reduced pressure at $55^{\circ} \mathrm{C}$ just prior to use. Water was distilled and deionized by a Milli-Q system from Millipore. BA (from Aldrich) and AIBN (from Fluka) were high quality products, used as received.

The total co-monomer concentration $\left(C_{\mathrm{T}}\right)$ and the cross-linker ratio $(C$, wt.- $\% \mathrm{BA}$ in the mixture of BA and VI) in the feeding mixture were $C_{\mathrm{T}}=40$ or 25 wt.- $\%$ and $C=2$ wt.- $\%$. Samples were identified in the following by the symbol PVIC $C_{\mathrm{T}}(C)$, i.e., PVI40(2) or PVI25(2). The aqueous solution of co-monomers and $\mathrm{AIBN}$ was sonicated at $60^{\circ} \mathrm{C}$ for 10 minutes and then the mould (cylindrical with flat bottom, $10 \mathrm{~mm}$ internal diameter and $2 \mathrm{~cm}$ height) was kept in an oven at $90^{\circ} \mathrm{C}$ for 24 hours. The hydrogels were removed from their moulds and cut into cylinders of about $1 \mathrm{~cm}$ height. The first compression measurement (post-polymerization test) was then carried out, with the specimen being weighed before and after. The hydrogel was then washed repeatedly with deionized water, replacing water from time to time and analysing it spectrophotometrically to detect the end of the extraction of soluble material, in particular, of residual monomer. Once cleaned, the specimen was immersed in the desired swelling medium for at least one week and the compression measurement was performed, with the specimen being weighed before and after. In some cases, the hydrogel was then swollen in a different medium to perform a new compression measurement. Otherwise, it was washed again with deionized water and dried in oven at $80^{\circ} \mathrm{C}$ for 24 hours to determine the weight of the xerogel.

Swelling measurements were performed gravimetrically following the method described in ref. $[5,11,12]$. The polymer volume fraction in the swollen gel, $v_{2}$, was determined from the mass of the swollen and the dry cylinder and xerogel densities which were measured at $26^{\circ} \mathrm{C}$ 
in the solid state with a Micrometrics 1305 Multivolume Helium Pycnometer. ${ }^{[11,12] .}$ pH measurements were carried out with a Corning 245 pHmeter, at room temperature. The degree of protonation $\alpha^{*}$ (to distinguish it from $\alpha$, the deformation of the swollen sample attained by compression) was determined by measuring the initial $\mathrm{pH}_{i}$ of the swelling media before immersion of the gel and the final value, $\mathrm{pH}_{\mathrm{f}}$, reached at equilibrium,

$$
\alpha^{*}=\frac{10^{-p H_{i}}-10^{-p H_{f}}}{C_{e f}}
$$

where $C_{\text {ef }}$ represents the effective gel concentration or moles of gel immersed by unit volume of the swelling medium.

Uniaxial compression measurements were performed on a Microtest (Spain) tensile testing machine at room temperature, using a load cell of $\pm 0.01 \mathrm{~N}$ sensitivity and $50 \mathrm{~N}$ maximum load. The compression probe consisted of a pair of parallel Teflon plates, wetted with a drop of water to minimize the appearance of shear stresses on the surface of the sample under uniaxial compression. Owing to the very small modulus of the samples, the initial length was determined as the separation between the upper and lower probes at which the load cell detected a signal above electronic noise. Such a length was measured with an optical ruler with a resolution of $\pm 1 \mu \mathrm{m}$. Once the upper probe was located over the surface of the sample, load was applied in successive steps of $0.02 \mathrm{~N}$ and the values of force and length were recorded in each step. Samples were weighed before and after compression measurements and no significant water evaporation was observed in any case.

\section{Theoretical Basis}

The shear modulus, $G$, was determined by linear regression from the dependence

$$
\tau=\frac{f}{A_{S}}=G v_{2}^{1 / 3}\left(\alpha-\alpha^{-2}\right)
$$

where $\tau$ is the applied stress in $\mathrm{Pa}, f$ is the applied force, $A_{\mathrm{S}}$ is the cross section of the undeformed swollen specimen and $\alpha=L / L_{0}$ is the relative deformation caused by compression of the length of the swollen sample along the direction of the stress $(L)$ with respect to the initial length $\left(L_{\mathrm{o}}\right)$ of the undistorted swollen sample.

The reduced force is defined as a function of the cross-section of the dry gel $A_{d}$ by

$$
\left[f^{*}\right]=\frac{f v_{2}^{1 / 3}}{A_{d}\left(\alpha-\alpha^{-2}\right)}
$$


and in terms of the cross section $\left(A_{\mathrm{S}}\right)$ of the undeformed swollen gel sample by

$$
\left[f^{*}\right]=\frac{f}{A_{S} v_{2}^{1 / 3}\left(\alpha-\alpha^{-2}\right)}=\frac{\tau}{v_{2}^{1 / 3}\left(\alpha-\alpha^{-2}\right)}
$$

The Erman-Monnerie theory ${ }^{[7,10]}$ expresses the reduced force in terms of the phantom modulus as:

$$
\left\lfloor f^{*}\right\rfloor=\left\lfloor f_{p h}^{*} \mid\left\{1+A_{\phi}^{-I} g(\alpha)\right\}\right.
$$

where $A_{\phi}=(1-2 / \phi)$ measures the functionality of the crosslinker ( $\phi=4$ for $\left.\mathrm{BA}\right)$ and $g(\alpha)$ is the strain-dependent part of the reduced force. It can be determined as a function of $\alpha, v_{2}, v_{2 \mathrm{r}}$ (the polymer volume fraction of the hydrogel in the reference state, which is commonly taken as the state of the network just after polymerization) and $\kappa_{\mathrm{i}}$, one of the two fitting parameters of the theory together with $\left[f_{\mathrm{ph}}{ }^{*}\right]$. The expression for $g(\alpha)$ also includes the magnitude $\Phi$ defined by Equation (6) and (7). Two different models of phantom network were considered in the theory, giving place to the formulation called the Constrained Chain or CC model, with

$$
\Phi=A_{\phi}^{2}\left(\frac{1}{3}+\frac{2}{3 n}\right)
$$

where $n$ represents the number of Gaussian subchains forming chains between knots, and to the Modified Constrained Chain or MCC model, with

$$
\Phi=A_{\phi}^{2}
$$

Fitting procedures were carried out using built-in Matlab optimization functions, which minimize the sum of the squared differences between experimental and calculated values of one (independent analysis) or several (global analysis) samples. The Gaussian character of network chains is one of the basic assumptions of the Erman-Monnerie theory.

\section{Results and Discussion}

Fig. 1 shows a representative uniaxial compression experiment with the typical plateau for the smallest range of deformation. ${ }^{[2,13-15]}$ It was attributed to imperfect geometry of the surface of the gel specimen in such a way that the usual correction was applied: the linear part in the graph of $\tau$ versus $\alpha-\alpha^{-2}$ was extrapolated to $\tau=0$ in order to determine the real initial $\alpha_{0}=L_{0}{ }^{\prime} / L_{0}$ and the corrected initial length $L_{0}$ ' with which new corrected $\alpha^{\prime}=L / L_{0}$ ' values were calculated and plotted as shown in Fig. 1. In the following only corrected values will be presented. Fig. 1 also shows the reproducibility of two consecutive loading experiments made on a sample just after polymerization. 
No significant hysteresis was observed for all the samples studied here after polymerization, the elastic modulus being about the same in consecutive loading and unloading experiments (Table 1). In contrast, large hysteresis was found for the same specimens swollen in water or acid aqueous solutions (Table 1) and hence, only loading experiments will be considered.

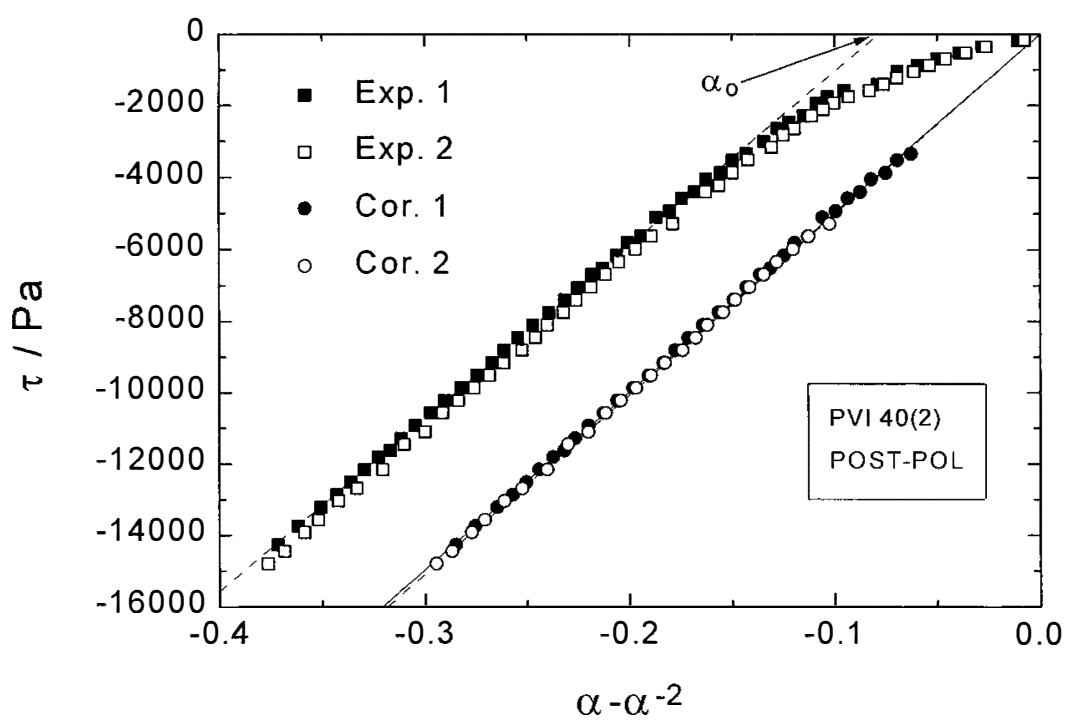

Fig. 1. Compression-strain measurements (Exp. 1 and 2, consecutive) and corrected data (Cor. 1 and 2) for PVI40(2) just after polymerization. The corrected initial length $L_{0}{ }^{\prime}$ was calculated from the real initial strain $\alpha_{0}=L_{0} \% L_{0}$ and the measured initial length $L_{0}$. By its side, $\alpha_{0}$ was determined from the intercept of the dashed line with the $\mathrm{X}$-axis.

Table 1. Shear modulus of several specimens of samples PVI40(2) and PV125(2) with different degrees of protonation $\left(\alpha^{*}\right)$, measured in loading and unloading experiments.

\begin{tabular}{cccccc}
\hline SAMPLE & $\begin{array}{c}\text { Swelling } \\
\text { Medium }\end{array}$ & $v_{2}$ & $\alpha^{*}$ & $\begin{array}{c}G(\mathrm{kPa})- \\
\text { Loading }\end{array}$ & $\begin{array}{c}G(\mathrm{kPa})- \\
\text { Unloading }\end{array}$ \\
\hline PVI40(2) & POST-POL. & 0.283 & - & 76.0 & 76.5 \\
Specimen 1 & $\mathrm{H}_{2} \mathrm{O}$ & 0.159 & - & 84.4 & 88.2 \\
& $\mathrm{H}_{2} \mathrm{SO}_{4} / \mathrm{H}_{2} \mathrm{O}$ & 0.0780 & 0.29 & 64.1 & 70.7 \\
PVI40(2) & $\mathrm{POST}-\mathrm{POL}$. & 0.273 & - & 60.1 & 62.3 \\
Specimen 2 & $\mathrm{H}_{2} \mathrm{O}$ & 0.138 & - & 84.8 & 79.2 \\
& $\mathrm{HCl} /{\mathrm{NaCl} / \mathrm{H}_{2} \mathrm{O}} 0.106$ & 0.13 & 63.9 & 79.7 \\
& $\mathrm{HCl} / \mathrm{H}_{2} \mathrm{O}$ & 0.0591 & 0.17 & 63.1 & 119.0 \\
PVI25(2) & $\mathrm{POST}-\mathrm{POI}$. & 0.128 & - & 21.6 & 21.4 \\
& $\mathrm{H}_{2} \mathrm{O}$ & 0.0873 & - & 28.6 & 29.5 \\
\hline
\end{tabular}


The first unexpected result was that $G$ values measured just after polymerization were, in all the specimens studied here, smaller than those of the same sample washed and swollen at equilibrium in pure water (Table 1). This behaviour was also observed in some previous reports. ${ }^{[2]}$ The theory has no explanation for this result since as swelling increases (decreasing $\left.v_{2}\right), G$ should decrease. Without taking into account the post-polymerization measurements (see Table 1) $G$ follows the expected trend, increasing upon increasing $v_{2}$.

Plots of the reduced force versus $\alpha^{-1}$ for two PVI samples synthesized with the same $C$ and two different $C_{\mathrm{T}}$ are compared in Fig. 2, as well as the best fit curves calculated with the Erman-Monnerie theory for the whole range of $\alpha$. Theoretical predictions with CC and MCC models overlap ${ }^{[7]}$ in the $\alpha$ range corresponding to compression $(\alpha<1)$ as observed in Fig. 2. Fits, both with CC and MCC models, were very good for flat plots such as that for PVI25(2) shown in Fig. 2, but for U shaped plots like that of protonated PVI40(2), it is not so. Upwards curvature is probably due to non-Gaussian effects associated with the large swelling degree and subsequent stretching of chains between knots. The theory translates that curvature in large values of $\kappa_{\mathrm{G}}$, which means that entanglements largely constrain the mobility of extended chains. Obviously, $\left[f_{\mathrm{ph}}{ }^{*}\right]$ is about equal to $G$ for flat plots and smaller than $G$ for samples with large $\kappa_{\mathrm{G}}$ having behaviour close to the affine limit.

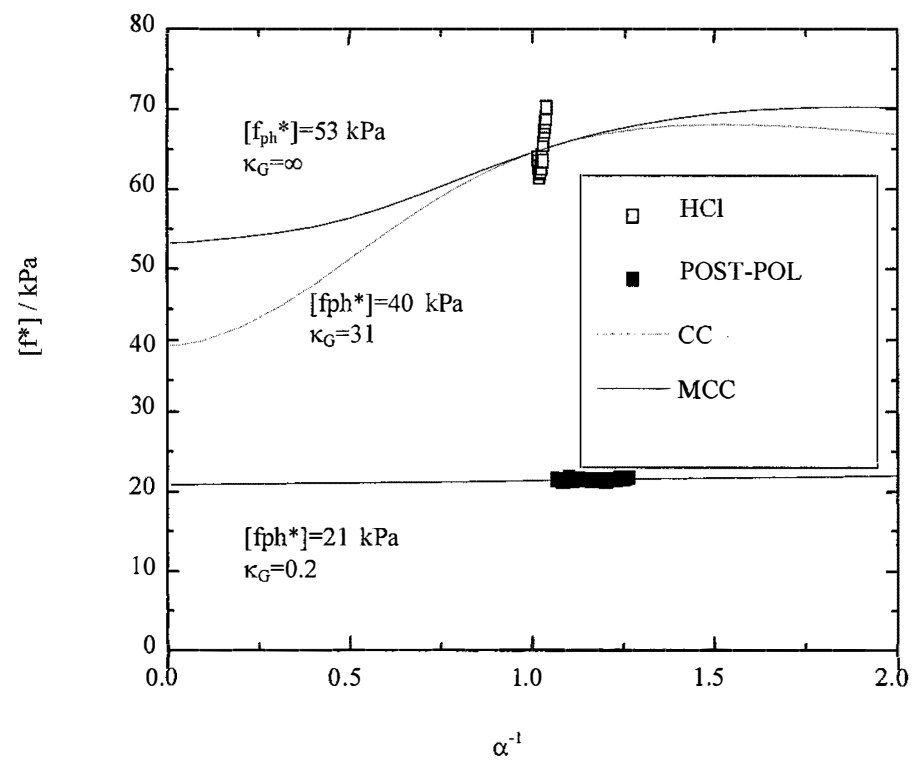

Fig. 2. Mooney-Rivlin type plots of the reduced force for PVI40(2) protonated with $\mathrm{HCl}$ and PVI25(2) measured just after polymerization. Curves correspond to best fits with MCC and $\mathrm{CC}$ models calculated in the whole strain range. 


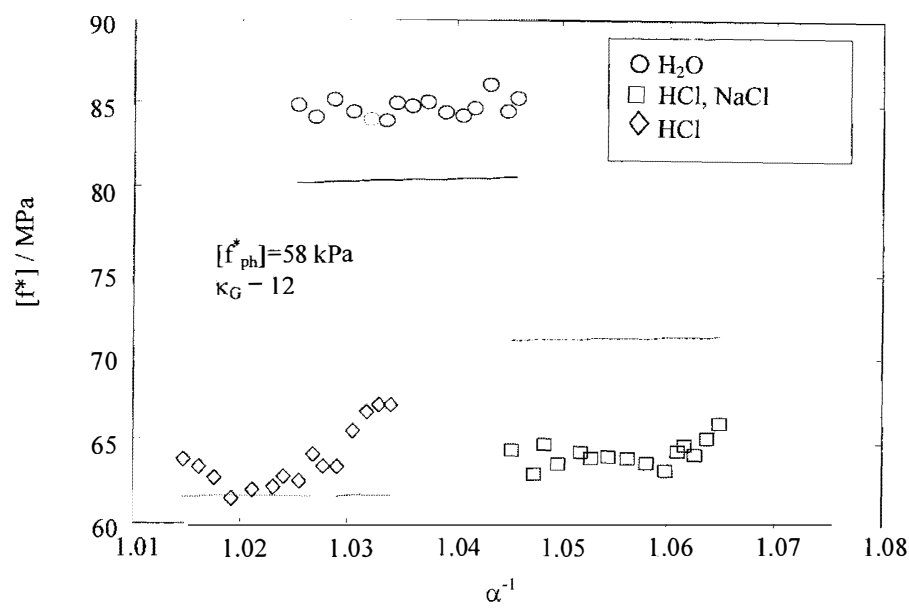

Fig. 3. Mooney-Rivlin type plots of the reduced force for PVI40(2) swollen in different media: pure water, $\mathrm{HCl}$ aqueous solution $\left(\mathrm{pH}_{\mathrm{i}}=2.5\right)$ and $\mathrm{HCl} /(\mathrm{l} M) \mathrm{NaCl}$ aqueous solution $\left(\mathrm{pH}_{\mathrm{i}}=2.5\right)$. Solid line represents the best fit with $\mathrm{MCC}$ model and global analysis of the three measurements.

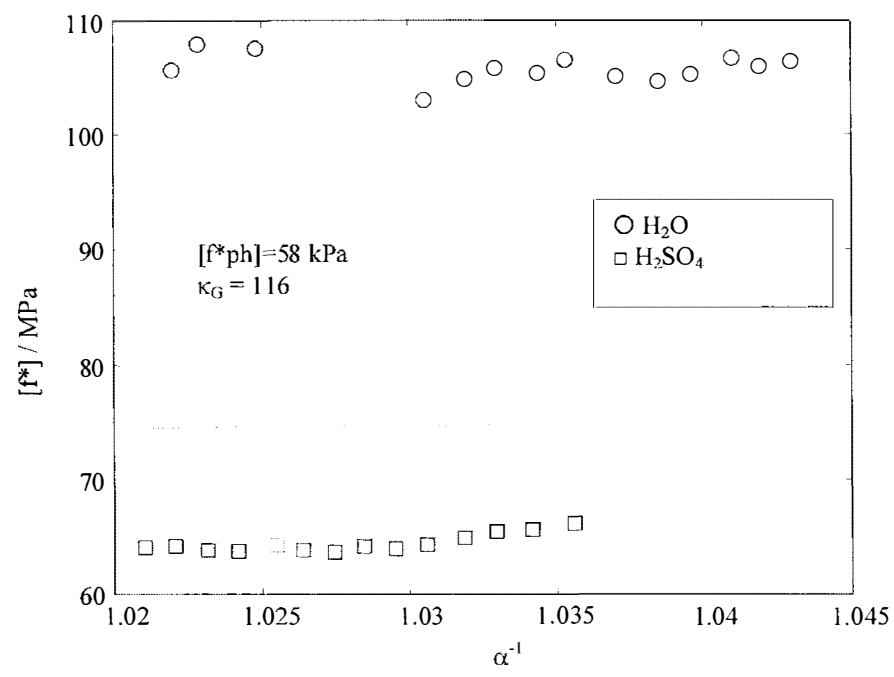

Fig. 4. Mooney-Rivlin type plots of the reduced force for PVI40(2) swollen in different media: pure water and $\mathrm{H}_{2} \mathrm{SO}_{4}$ aqueous solution $\left(\mathrm{pH}_{\mathrm{i}}=2.5\right)$. Solid line represents the best fit to $\mathrm{MCC}$ model and global analysis of the two measurements.

Figure 3 and 4 compare the behaviour of neutral and ionic (protonated) hydrogels. The global analysis of measurements made on both types of hydrogel yields good enough results and it is 
remarkable that two different specimens show the same fitted value of $\left[f_{\mathrm{ph}}{ }^{*}\right]$ (Fig. 3 and 4).

This should be expected, since both were synthesized with the same $C$ and $C_{\mathrm{T}}$ in the feeding mixture. The yield of the polymerization is, however, different as reflected in the slightly different $v_{2 \mathrm{r}}$ values ( $v_{2}$ post-pol in Table 1) and usually, this causes small differences in the resulting cross-link density. Both specimens show large $\kappa_{\hat{j}}$ values (Fig. 3 and 4), meaning that fluctuations are almost frozen as in the affine model.

In order to explain that samples protonated with $\mathrm{H}_{2} \mathrm{SO}_{4}$ show smaller degrees of swelling than those protonated with $\mathrm{HCl}$ with the same degree of protonation, it was suggested ${ }^{[5]}$ that divalent anions (as for $\mathrm{H}_{2} \mathrm{SO}_{4}$ ) might induce the formation of ionic cross-links. But no evidence for that additional cross-link density was observed in compression measurements, except, perhaps, for the much larger $\kappa_{\mathrm{G}}$ value of the specimen protonated in $\mathrm{H}_{2} \mathrm{SO}_{4}$.

\section{Conclusions}

The compression modulus of PVI hydrogels was determined for swollen samples in several media. Just after polymerization, the modulus was lower than expected, taking into consideration the polymer volume fraction in that state. Nevertheless, measurements just after polymerization yielded flat Mooney-Rivlin plots whereas protonated samples give $U$ shaped plots denoting non-Gaussian chains between knots. In spite of such non-Gaussian character. global analysis of measurements made on neutral and protonated hydrogels using the ErmanMonnerie theory was possible and yielded consistent values of the two fitting parameters.

\section{Acknowledgements}

Financial support from DGI (Spain) under grants BQU2000-0251 and MAT2000-0391-P4-02 is gratefully acknowledged.

[1] J. Baselga, I. Hernández-Fuentes, I. F. Piérola, M. A. Llorente, Macromolecules 1987, 20, 3060.

[2] O. Okay, S. Durmaz, Polymer 2002, 43, 1215.

[3] T. P. Davis, M. B. Huglin, Makromol. Chem. 1991, 189, 195.

[4] A. Ikehata, H. Ushiki, Polymer 2002, 43, 2089.

[5] M. J. Molina, M. R. G. Antón, I. F. Piérola, Macromol. Chem. Phys. 2002, 203, in print.

[6] G. Nisato, S. J. Candau, in: "Polymer Gels and Networks", Y. Osada, A. R. Khokhlov, Eds.Marcel Dekker, New York 2002, p. 131-162.

[7] B. Erman, L. Monnerie, Macromolecules 1989, 22, 3342.

[8] F. Fontaine, C. Morland, C. Noel, L. Monnerie, B. Erman, Macromolecules 1989, 22, 3348.

[9] F. Fontaine, C. Noel, L. Monnerie, B. Erman, Macromolecules 1989, 22, 3352.

[10] B. Erman, L. Monnerie, Macromolecules 1992, 25, 4456.

[1 1] J. Valencia, I. F. Piérola, Eur. Polym. J. 2001, 37, 2345.

[12] J. Valencia, I. F. Piérola,. J. Appl. Polym. Sci. 2002, 83, 191.

[13] A. Gutowska, Y. H. Bae, H. Jacobs, J. Feijen, S. W. Kim. Macromolecules 1999, 27, 4167.

[14] E. C. Muniz, G. Geuskens, Macromolecules 2001, 34, 4480.

[15] C. Sayil, O. Okay, Polymer 2001, 42, 7639. 